\title{
Lane Departure Detecting with Classification of Roadway Based on Bezier Curve Fitting Using DGPS/GIS
}

\author{
Ihn-Sik WEON, Soon-Geul LEE*, Soo-Ho WOO
}

\begin{abstract}
Lane departure warning system plays an important role in safety driving by detecting a departure from a lane that is inadvertently operated on the driving trajectory. This paper suggestion detection algorithm when a vehicle departs lane boundary using GIS based on DGPS in the whole roadways. Lane segments obtained from the GIS are calculated their relative distances based on the vehicle position. Lane segments consist of consecutive straight lines and have a steady numerical error of design. In the curved section, the numerical error is bigger due to the characteristics. Accurate information about lane segments is required to reduce errors. Bezier curves are one way to extract lane segments from a curved section. The proposed lane departure algorithm is processed in two ways according to the lane type. Firstly, roads should be classified as lane type with straight and curved sections. Intersection points (IP) algorithm can easily classify the curve segments. Classified lane segments handle arithmetic relative distances for each algorithm. The lane segment of the base boundary, which is a straight lane section, has a virtual line based on the requirements of ISO 17361. The overlap area, consisting of a curve lane section and a Bezier curve, calculates the departure distance through the continuity of the driving characteristic and determines the lane departure from the curved roadways. To verify the proposed algorithm, the lane departure test led to two lane departures on each roadway. The comparison between visual sight and the departure alarm shows the driver within 0.1 second.
\end{abstract}

Keywords: bezier curve; classification roadway; DGPS/GIS; intelligent function; loT; lane departure; precision localization

\section{INTRODUCTION}

Fusion technology of multi-sensor for vehicle positioning has been developed at a rapid pace over the past decades [10]. Accuracy positioning for vehicle is an important key that has improved performance of safety technology and helped to safe driving correspond with environment. Intelligent vehicle which comprises electronic, electromechanical, and electromagnetic system is required to have robust safety controls and to provide precision repeatability functions. The one of intelligent functions for vehicle is the driverless vehicle, which is capable of sensing its environment and navigating without human intervention [12].

Performance property of a collision avoidance system based on a vision camera is to track lane makers on the road for detecting the lane departure of the vehicle. An effective system needs to detect the possibility of collision when the vehicle is departing a lane and to determine initial time to warn the driver early enough to return [4]. The key elements of detecting lane departure are that it uses concept known as warning line to determine the vehicle is within a lane. An avoidance of collision algorithm is required warning line to prevent accidents.

Warning line is defined by ISO 17361:2007. There are two types of warning line, one is the earliest warning line and the other is the latest warning line. The former is the innermost limit of the warning threshold and the latter is the outermost limit of the warning threshold. Warning line is available to confirm start and end when a vehicle departs a lane (2007). Location of actual warning line is rarely given to intelligent systems. Moon (2012) defined the position of the warning line with respect to the lane boundary which was obtained by GIS [7]. Jung (2009) has attracted integrating both functions of LKS+ACC system with embedded vision [2].

The map of information include road type is one of the key that make it possible to the problem of vision camera [4]. Although precision positioning and mapping techniques from the road shape have not yet been fully certified, below studies have proceeded actively. Southall (2001) described a new system for estimating a road shape by combining information of a single camera and heading angle of the vehicle [13]. Moon (2012) has implemented lane departure warning (LDW) system based on the concept of warning line that depends on DGPS error [9].

Lane boundary of segment obtained from GIS consists of coordinate and this segment is built up of straight line. Recent reporters suggest that the lane boundary of segment used to be classified a straight- and curved-line segment based on same direction of lane. The straight line can be separate to straight and curved section by feature variable with relatively angle. The feature variable of intersection is calculated by distance and a range of angle from continuous segments. The lane boundary of segment has been simplified by fitting which from separated lane type, and the segments make for quick arithmetic that could be used in a lane departure system [3, 18].

In this research, we can find the intersection point between the lane types (straight and curve) with computation result comparable to that of the correlation of gradient. It is essential to separate whether a curved lane or not and to calculate the gap before the lane departure happens. The line segment with curvature generates coordinate values of curved feature by Bezier curve using 3 points of referenced curve line [11]. The lane departure algorithm should be also applied separately in the straight and curved lane for drivable path. The velocity of driving, its location, and segments of lane boundary and vehicle width are utilized detecting algorithm to reduce error. These requirements determine location of earliest warning line based on specification of positioning sensors and often involve estimated trajectory to determine quick and robust a warning signal. Above all, the algorithm can help to reduce arithmetic operation time, to make a decision and a warning.

\section{CLASSIFICATION FOR THE LANE BOUNDARY SEGMENT}

Lane boundary is on a paved lane to provide guidance and information to drivers. It used to minimize confusion and uncertainty for driving roadways. The boundary lane of segment has coordinate obtained from GIS, but it is 
difficult to maintain their marking as well as updating the lane boundary of segment. To maintain the boundary lane of segment, Kim suggested basis concept to update $X Y$ coordinates as useful information for detecting safety drive [3]. The coordinate data for specific lane segments (straights, curves) is adapted to discriminate a departure status by calculating a gap with the vehicle positioning. A part of the evidence for improvement of a departure system is prepared to use the database of map obtained by GIS. The robust resulting of detection is started from seeking an intersection point between a straight lane and a curved lane.

It is necessary to analyse alignment radius of curvature for efficient intersection of the straight lanes. The characteristic of correlation coefficient which the gradient of continuous points can extract the intersection features by using boundary lane of the map tries to implement on GIS. In the case of Moon, which verified the intersection points for classification of lane types between straight and curved roadway [8].

The intersection point for the longest curved/straight section is able to extract by computing Eqs. (1) to (3) based on the demonstration of Moon for utilizing lane segments [6].

By using extracting criterion with computation as defined in Eqs. (1) to (3), the computation is to find a inflection point along path of the lane. To find inflection point, computation value is expressed the accumulated grade from start point to limited point. This accumulated grade of value is most reliably determined by 2 -ways using first point and previous point.

The subscript $f$ of Equation is the first point in the lane segments, the subscript $p$ is a previous point based on the $k$-th point $[16,17]$.

$$
\begin{aligned}
& \beta_{(p, f)}^{k}=\left[2 \cdot \arctan \frac{d y_{(p, f)}^{k}}{\sqrt{\left(d x_{(p, f)}^{k}\right)^{2}+\left(d y_{(p, f)}^{k}\right)^{2}}+\left(d x_{(p, f)}^{k}\right)}\right],(-\pi, \pi) \\
& M_{(p, f)}^{k}=\frac{\sum_{k=1}^{n} \beta_{(p, f)}^{k}}{n} \\
& C_{(p, f)}^{k}=\frac{\sum_{k=1}^{n}\left(\beta_{(p, f)}^{k}-M_{(p, f)}^{k}\right)}{n}
\end{aligned}
$$

Where $\left(d_{x}, d_{y}\right)$ is the distance difference value between two points complied given condition. Gradient of lane segments $\left(\beta_{f}^{k}, \beta_{p}^{k}\right)$ is given by Eq. (1). We assume $\left(M_{f}^{k}, M_{p}^{k}\right)$ is an average value for gradient of lane segment as shown Eq. (2). $\left(C_{f}^{k}, C_{p}^{k}\right)$ is the correlation coefficient of the lane segment. Calculated $\left(C_{f}^{k}, C_{p}^{k}\right)$ is used as a criterion by conversion through the distinguishing point extraction algorithm.

Correction coefficient are utilized to find intersection points and classify a roadway type with a straight or curved. The extracted result of intersection point using Roadway Characteristic Conversion Coefficient with limited criteria area. The intersection point between straight and curved section has a criterion that is crossing angle between consecutive lines [6].

\subsection{Expression of Line: Straight Segment}

We can decide the comparative location of the vehicle and lane by making the extracted coordination value of boundary lane in the straight-lane segment like the above one as the linear equation.

The error of the recommended lane is declined by decision of the lane type, also it has advantage for the comparative decision in the quick calculation when the high-speed situation.

Each extracted point of boundary line should be expressed and by reference center-lane based on tworoadways.

$$
\begin{aligned}
& L_{c n}=\left(X_{c n}, Y_{c n}\right), L_{c n+1}=\left(X_{c n+1}, Y_{c n+1}\right) \\
& P v_{i}=\left(X v_{i}, Y v_{i}\right), P v_{i+1}=\left(X v_{i+1}, Y v_{i+1}\right)
\end{aligned}
$$

Where $P v_{i}$ is the current position, $P v_{i+1}$ is next position. Position of lane will be executed $n$-times, but the location of vehicle will performed in accordance with $i$-time of DGPS time synchronization.

To classify a straight segment requires simply calculating each boundary lane by connected point at 7 meter intervals, as in Fig. 2. When the points was determined continuously by two points of boundary lane, $L_{c n}, f\left(L_{c n}\right)$, and their derivatives are determined using Eq.(6).

$$
f^{\prime}\left(L_{c n}\right) \approx \frac{f\left(L_{c n-1}\right)-f\left(L_{c n}\right)}{L_{c n-1}-L_{c n}}
$$

Fig. 1 shows extracted lanes of straight segment using 2 points $L_{c n}$ and $L_{c n+1}$ based on center boundary lane. The right $L_{\mathrm{R} n}$ of the lane, the left side of the road was referred to as the $L_{\mathrm{L} n}$ subscript from the traveling direction with respect to the center of boundary lane. Set the local reference frame $(X s$, $Y s$ ) indicates a vehicle orientation angle $\gamma$ by the global reference frame transformation.

A vehicle localization of coordinate data is refined in the local reference a $\left(X v_{i}, Y v_{i}\right)$. This position are calculate with lane boundaries segments above defined to measure their relative gaps. The gap is shown in the $D^{\mathrm{R}}$ of the right boundary lane, $D^{\mathrm{L}}$ of the left boundary lane.

\subsection{Expression of Line: Curve Segment}

One important factor in design of a curved road is the radius of curvature based on standard speed. Points extracted from line data of GIS can be straightened from which there is a curved lane segment, but they has tended to require many points to reduce distance error. Thus, we demonstrated here a curved lane from a quadratic Bezier curve. To show an inactively curved road Fig. 2 that denotes a fitting line based on a quadratic Bezier curve that combines two consequent of lines by linking points of line. This is used to have feature that affect curved shape by controlling vertex more than an order of curve equation [11]. 


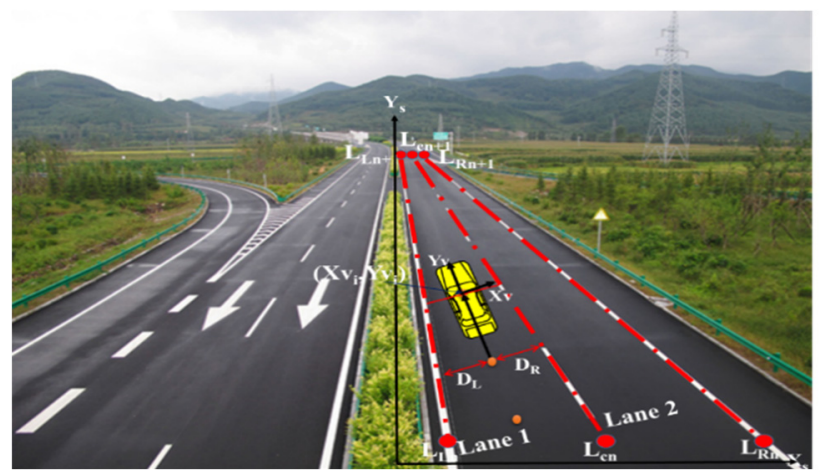

Figure 1 Straight lane segment \& update of GIS line

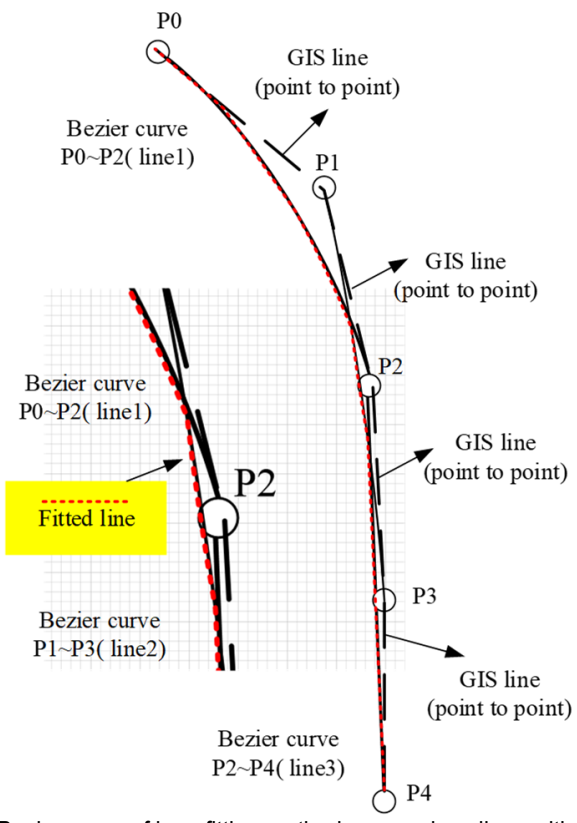

Figure 2 Bezier curve of lane fitting method: comparison lines with fitted lane of GIS [15]

Hence, the control point $\mathrm{P}_{N}$ guarantees the expression of the curve lines. Resulting information of curve line can be obtained with the second order Bezier curve equation, where $B(N)$ of position of $N$ step has setup interval 0.01 steps between 0 to 1 second, and $\mathrm{P} 0, \mathrm{P} 1$, and $\mathrm{P} 2$ refer to start, control, and end point, respectively, finding a dividing point for line segment P0, P1 in $t$, seconds.

These dividing points transpose and repeatedly control with a dividing point for line segment P1P2.

Bezier curve fitting used to make a curve line by 3points P0-P1-P2, and their set of curve line used arithmetic operations for detecting a lane departure during driving at the curve lane. Marginally view of Fig. 2 is shown originally line points that expressed a dashed line of black color, and resulting points of Bezier curve is expressed straight line of the black color. Dotted line of red color is expressed fitted line through overlap section. Points of curved section are extracted by above roadway type classification technique.

\section{DETECTING ALGORITHM FOR LANE DEPARTURE}

For operation of a lane departure system, a DGPS receiver should receive data that is relatively denoted with respect to the line from GIS. The type of the current lane can be classified with the information and lane departure can be detected with lateral velocity. The criteria values for classification and the threshold value for lane departure are obtained at the vehicle speed $1.0 \mathrm{~m} / \mathrm{s}$ according to ISO 17361: "Test Procedure" [1].

Those criteria values shall be able to give a parameter under radius of curvature and vehicle speed, but give a visible lane marking, rate of departure, time to line crossing, warning issue point. Also, its repeatability test has a rate of departure condition to achieve within group according to the rate-of-departure tolerance given test report.

\subsection{Detecting of Departure for Boundary Line: Straight Lane}

When the velocity suddenly changes more than $1.0 \mathrm{~m} / \mathrm{s}$ or its changing rate is rapid, the driver receives a warning of careless driving, regardless of departure status.

High speed of vehicle would be remained 20 to $40 \mathrm{~cm}$ by depending on error of sensor from warning line to lane boundary. Then, vehicle used to spend time to cross the lane boundary within 1 seconds.

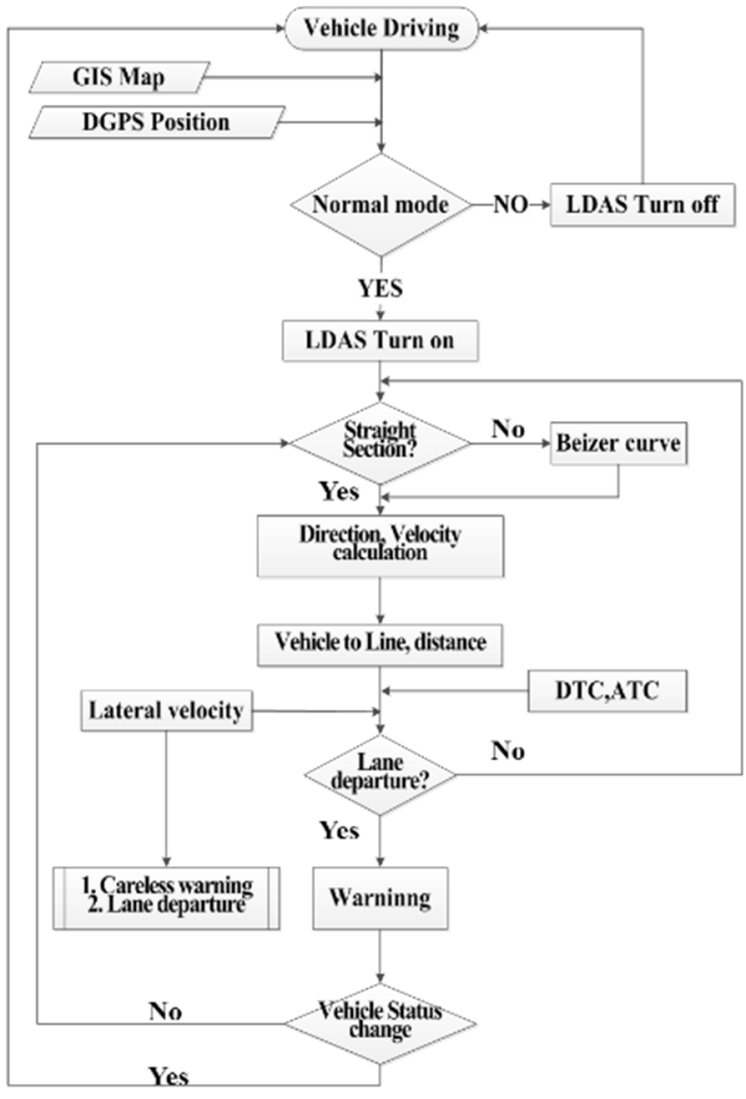

Figure 3 Lane departure detecting step

DTC and ATC are in need to find a lane departure as path prediction in advanced. Fig. 3 shows the concept of DTC and ATC. The path prediction was affected by the former pathway and speed. The prediction point that have passed a period is cross the warning line, and then the system is a warning to driver. By considering that the reaction rate of a person, a period is 0.5 seconds. The prediction point consisted of $n$-th points by same direction as the previous progress. Fig. 4 is indicative of two-virtual path prediction as departure status or not. Although the estimated point $P v_{i}$ is not crossing a boundary line, prediction point by DTC and ATC is a warning. Prediction position of the vehicle $P v_{i}$ are estimated by combining 
position, vehicle speed, lateral speed, and heading angle, as shown in Fig. 4. $P v_{i}$ has been progressed to the path of $\hat{P} v_{i}$ or $\hat{P} v_{i}^{\prime}$ according to previous points $P v_{i}$ and $P v_{i-1} . \theta_{k}$ and $\theta_{k}^{\prime}$ are the crossing angle when the predicted path by various parameters intersects the boundary of line and vehicle path [14]

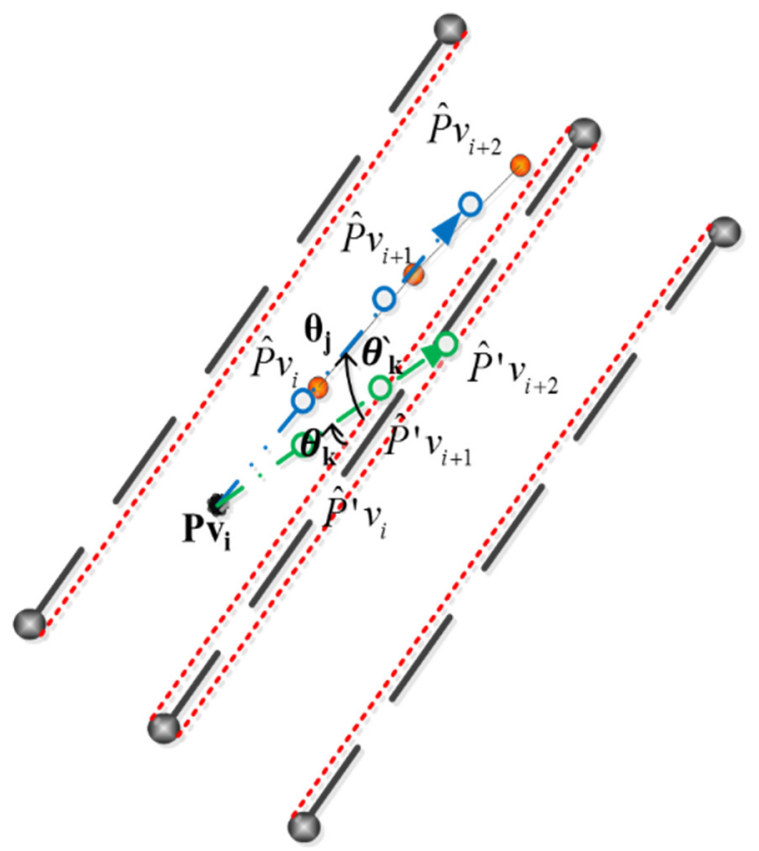

Figure 4 Detecting of lane departure theory

$P v_{i}\left(X v_{i}, Y v_{i}\right), \hat{P} v_{i}\left(\hat{X} v_{i}, \hat{Y} v_{i}\right)$

$\hat{P} v_{i}=\left[\begin{array}{l}X v_{i} \\ Y v_{i}\end{array}\right]+\left[\begin{array}{c}\cos \theta_{k} \\ \sin \theta_{k}\end{array}\right] V v_{k} \cdot T_{s}$

$\theta_{k}=360^{\circ}+\arctan \left(\frac{Y v_{i}-Y v_{i-1}}{X v_{i}-X v_{i-1}}\right)$

$T_{s}=t_{i}-t_{i-1}$

$P v_{i}$ of presented position used to predict the next point by previous position of $P v_{i-1}$ where came from. The driver should be warned within one second to control the vehicle according to cognitive response in the event of lane departure.

$\hat{P} v_{i}$ and $\theta_{k}$ are determined Eqs. (7) to (10). They are displacements taken between timestamps $T_{s}$ in the vehicle's path. $X v_{i}$ is longitudinal position (along the satellite's axis) and $Y v_{i}$ is lateral position. $T_{s}$ is $0.05 \mathrm{~s}$ the same as the GPS time. The distance between the vehicle and the warning line $D_{k}$ is given by Eq. (11). We assume $D_{k}$ is the sum of each $D_{i}$, since the trajectory is predicted for $n$-times. Number of time interval is $n$ until a prediction point crossing the warning line.

$D_{k}=\sum_{k=1}^{n} \frac{\left|a_{i}\left(\hat{X} v_{i}\right)+b_{i}\left(\hat{Y} v_{i}\right)+c_{i}\right|}{\sqrt{a_{i}^{2}+b_{i}^{2}}}$
$c_{i}=X_{c n} \cdot\left(X_{c n+1}-X_{c n}\right)-X_{c n}\left(Y_{c n+1}-Y_{c n}\right)$

$\frac{\sum_{k=1}^{n} D_{k}}{n}<d$

The warning line of coordinate is expressed $L_{c n}$ based on center-line and split a vector into two-components as $X_{c n}, Y_{c n}$.

Moon et al. [12] have reported that a standard departure distance is dependent on ability of DGPS and lane width, which are now applied in this research. Their rate constant, $\mathrm{d}$, will be made solvable reference distance between prediction position of vehicle and the warning line as Eq. (13).

Fig. 3 shows the full procedure for advanced lane departure algorithm, and is given determined factors by DTC, ATC and lateral velocity.

With these considerations, an advanced method can be assumed for the efficiency of departure warning in real-time combining lane of GIS data and departure algorithm. Eq. (17) considered width of vehicle and lane, where $w$ is lane width and $L$ is a width of vehicle side, and $\left(D^{\mathrm{R}}, D^{\mathrm{L}}\right)$ is the distance of each axis based on vehicle direction.

$$
\begin{aligned}
& D^{\mathrm{R}, \mathrm{L}}\left\langle\left(\frac{w}{2}-\frac{L}{2}\right)+d, d \in(0,0.25)\right. \\
& D^{\mathrm{R}}+D^{\mathrm{L}}=w
\end{aligned}
$$

The value of distance $\left(D_{k}^{\mathrm{R}}, D_{k}^{\mathrm{L}}\right)$ from prediction path is computed as follows:

$$
D_{k}^{\mathrm{R}, \mathrm{L}}=\sum_{k=1}^{n} \frac{\left|a_{i} P_{k}+b_{i} P_{k}+c_{i}\right|}{\sqrt{a_{i}^{2}+b_{i}^{2}}}
$$

The coordinate values of Left and Right are respected to the vehicle coordinate. For fast processing during driving of vehicle, the left warning line is defined as a straight line passing both $P v_{i}^{\mathrm{L}}$ and $P v_{i+1}^{\mathrm{L}}$. The right warning line is defined as one passing both $P v_{i}^{\mathrm{R}}$ and $P v_{i+1}^{\mathrm{R}}$ . If the position of DGPS cross over these warning lines, then it can be determined that lane departure has occurred. Furthermore, the gaps between the vehicle and the warning line can be calculated by Eqs. (19) and (20).

$$
P v_{i}^{c}\left(X v_{i}^{c}, Y v_{i}^{c}\right) \text { and } P v_{i}^{\mathrm{L}}\left(X v_{i}^{\mathrm{L}}, Y v_{i}^{\mathrm{L}}\right) \text { of relative value }
$$

from the warning line are calculated in Eqs. (17) to (18). The vehicle coordinate $(X v, Y v)$ is the coordinate frame that is attached to the DGPS, and the $Y v$-axis is parallel to the lane.

The Present position $P v_{i}\left(X v_{i}, Y v_{i}\right)$ of vehicle and prediction position of $\left.\hat{P} v_{i}^{c}\right|_{i} ^{k}\left(\hat{X} v_{i}^{c}, \hat{Y} v_{i}^{c}\right)$ make an angle to collision as $\left.\varphi\right|_{i} ^{c}=\theta_{k}$. These parameters are used for calculation of distance $D_{k}$ for warning decision. 


$$
\begin{aligned}
& X v_{i}^{\mathrm{L}}=\frac{X v_{i}^{\mathrm{L}}+\left(X v_{i}-Y v_{i}^{\mathrm{L}}\right)}{\left(Y v_{i}^{\mathrm{L}}-Y v_{i+1}^{\mathrm{L}}\right)\left(X v_{i}^{\mathrm{L}}-X v_{i}^{\mathrm{L}+1}\right)}, Y v_{i}^{\mathrm{L}}=Y v_{i} \\
& X v_{i}^{c}=\frac{X v_{i}^{c}+\left(X v_{i}-Y v_{i}^{c}\right)}{\left(Y v_{i}^{c}-Y v_{i}^{c+1}\right)\left(X v_{i}^{c}-X v_{i}^{c+1}\right)}, Y v_{i}^{c}=Y v_{i}
\end{aligned}
$$

\subsection{Detecting of Departure for Boundary Line: Curve Lane}

If the vehicle drives on a curved lane, the gaps of departure is not correct by curvature features. Because $X Y$ coordinate of curved lane segments has error factors as like transformation, curvature approximation and design road.

The new coordinates of lane obtained by the Bezier curve method of transformation method is compared with the vehicle position while the curve section. Bezier method can be generated a hundred-coordinates within three markers of lane information. That is lane markers exist a group by 0.01 interval.

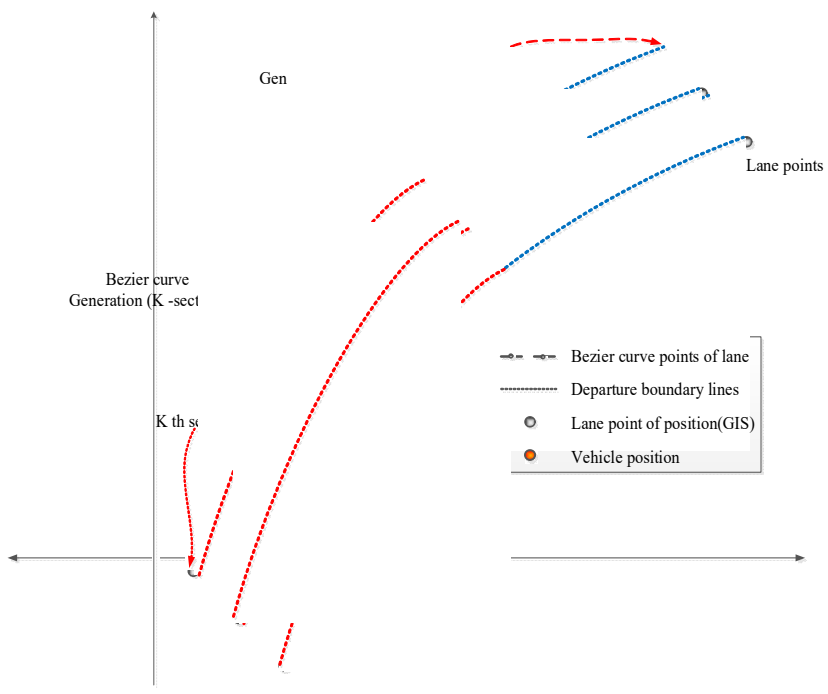

Figure 5 Diagram of departure decision of a curved road

As shown Fig. 5, there are 4-circle points as lane marker every lane course, and 4-semisolid squares for vehicle of trajectory. $k^{\prime}$-th section and $(k+1)$-th section was overlapped as same section. The relative distance based on vehicle between a $k$-section at $\left(X^{k+1}, Y^{k+1}\right)$ and an adjacent $k^{\prime}$-th section an $\left(X^{k^{\prime}}, Y^{k^{\prime}}\right)$ was calculated by vehicle position.

They have new coordinates of curve lane by Bezier method, accordingly distance of the calculation result between vehicle and lane markers must utilize either those lane section.

Algorithm has made to examine the potential sources of error in the arithmetic. Then, distance of $D_{k}$ used to be choice a short length in the reference value. In order to estimate the comparison with consecutive values the information of new lane created by Bezier method, it must be in within $5 \mathrm{~cm}$ of the difference from previously distance of $D_{k}$. Set of coordinates is a select elements showing a formula selected on the basis of the above conditions Eq. (19). Fig. 6 is showing the value of the distance relative to the lane during a normal driving at curved lane. The solid line is the result of $k$-th section transformed by Bezier curve and dotted line is the $k^{\prime}$-th section, which is a comparison of the values of the differences in the lane coordinate set of the section was measured. Test orders are shown as different color as black and red.

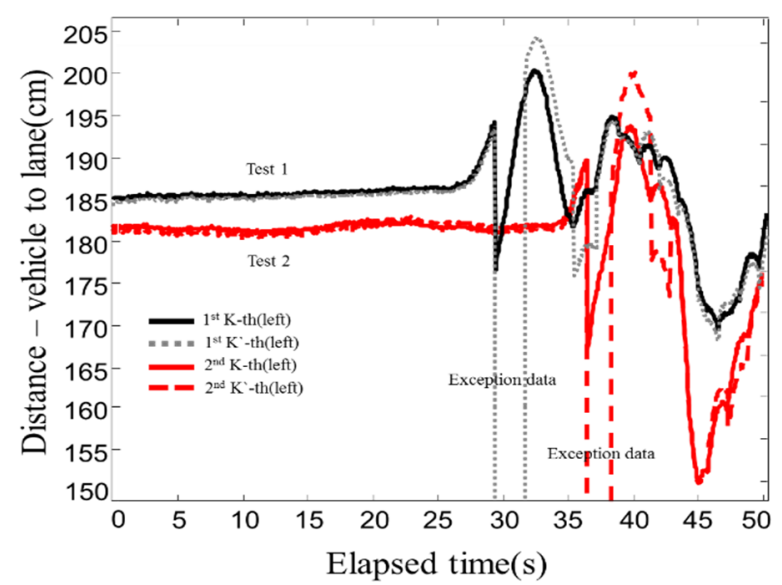

Figure 6 Different gaps when the vehicle driving a straight in curved road with Bezier curve markers

The value of the relative distance associated with the arithmetic operation, choose a small value, but the status information that is displayed in a large gap from the previous location in accordance with the driving, that does not appear when the vehicle drive naturally. Therefore, to be configured as shown in Eq. (19) as shown distance values like the previous state.

As can be seen Fig. 6, as in the case of 45 seconds, a distance difference when moving phenomenon of the traveling vehicle when traveling straight is continuously varied is displayed as well. However, 35 to 40 seconds and 30 seconds near can confirm that the distance values of the two-lane information are displayed in a large gap.

$$
\left[\begin{array}{cc:c}
X_{11}^{k} & Y_{11}^{k} & P_{1}^{i} \\
X_{12}^{k} & Y_{12}^{k} & P_{2}^{i} \\
X_{13}^{k} & Y_{13}^{k} & P_{3}^{i} \\
\hdashline \ldots & \ldots & \ldots \\
X_{1 n}^{k} & Y_{1 n}^{k} & P_{n}^{i}
\end{array}\right]=\left\{\begin{array}{l}
{\left[\begin{array}{ll:l}
X_{11}^{k+1} & Y_{11}^{k+1} & P_{1}^{i} \\
X_{12}^{k+1} & Y_{12}^{k+1} & P_{2}^{i} \\
X_{13}^{k+1} & Y_{13}^{k+1} & P_{3}^{i} \\
\hdashline \ldots & \ldots & \ldots \\
X_{1 n}^{k+1} & Y_{1 n}^{k+1} & P_{n}^{i}
\end{array}\right]} \\
{\left[\begin{array}{cc:c}
X_{11}^{k^{\prime}} & Y_{11}^{k^{\prime}} & P_{1}^{i} \\
X_{12}^{k^{\prime}} & Y_{12}^{k^{\prime}} & P_{2}^{i} \\
X_{13}^{k^{\prime}} & Y_{13}^{k^{\prime}} & P_{3}^{i} \\
\ldots & \ldots & \ldots \\
X_{1 n}^{k^{\prime}} & Y_{1 n}^{k^{\prime}} & P_{n}^{i}
\end{array}\right]}
\end{array}\right\}
$$

\section{RESULT AND DISCUSSION}

First of all, this is the experiment to drive without bouncing the lane. The test conditions are obtained from ISO 17361:2007, which is preferred that to verify the lane departure algorithm to check whether the error behaviours of straight driving.

For test scenario, system is composed of differential geography positioning system (DGPS) with the Radio Technical Commission for Maritime Services of message from base station using communication for wireless access in vehicular environments (WAVE) as shown Fig. 7. The 
measurement precision for static status is shown under 20 $\mathrm{cm}$ normally, and vehicle width is $180 \mathrm{~cm}$. Data processing with algorithm is based on visual $\mathrm{C}$ program being associated with former study, (Moon et al., 2013).

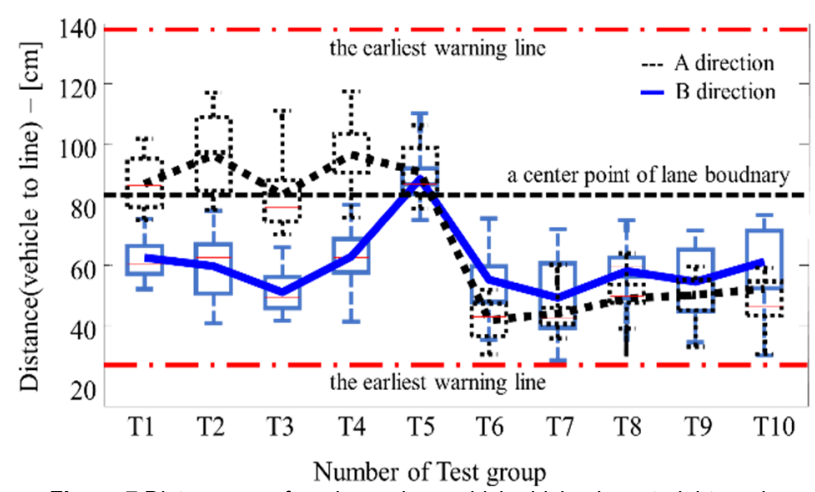

Figure 7 Distance gap from lane when vehicle driving in a straight road

\subsection{Detecting of Departure for Boundary Line: Curve Lane}

We demonstrated false alarm test to verify algorithms. Despite of different the habits of the traveling vehicle, the result of straight traveling shows location at the safety zone within an alternate long and short dashed line with red color as shown in Fig. 8.

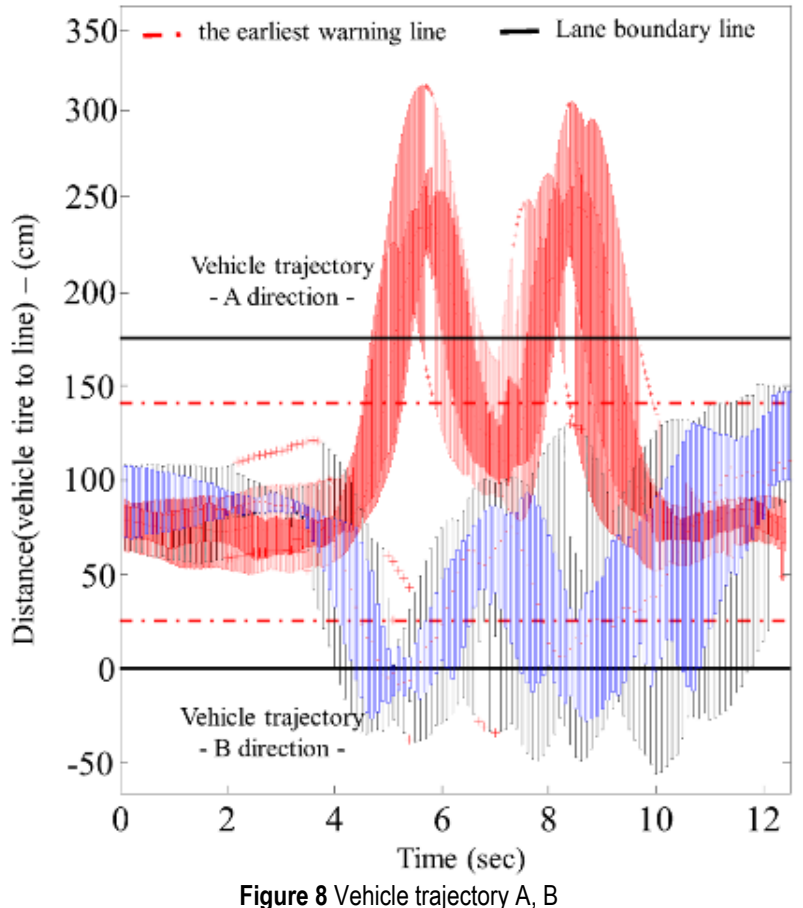

This results means the algorithm set a reliable operation in performance. Definition the criterion of boundary lines, which one of left side sets $0 \mathrm{~cm}$ and the other side, is $139 \mathrm{~cm}$ as limited distance with the earliest warning line.

After algorithm is operated well without false alarm at above straight driving test, mainly test of lane departure from straight/curved roadway has performed to try departing the lane boundary at two-times.

For performing over a period of two times, after vehicle has tried to depart lane boundary at first time, vehicle preferentially returns the lane for eliminating warning alarm. Fig. 9 is showing the result of experiment when a vehicle driving straight roadway.

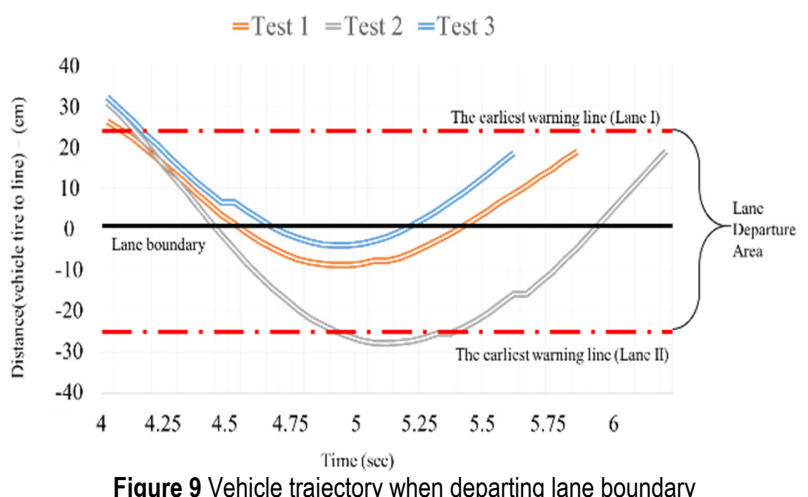

The red and blue blocks of distribution is average variance of distance between vehicle tire and lane boundary line segments. The distance has computed by above method consideration of vehicle width. In normal driving between 0 to almost $4 \mathrm{~s}$, the reported vehicle is at the center in the lane section, which is within 0.7 and 0.9 $\mathrm{m}$ based on one side of the lane boundary. After that, blue and red blocks has demonstrated of departing lane boundary as left and right side of the earliest warning line with green dotted line. The path of vehicle positioning shows a returning status according to test scenario between 6 to $8 \mathrm{~s}$ and after $10 \mathrm{~s}$.

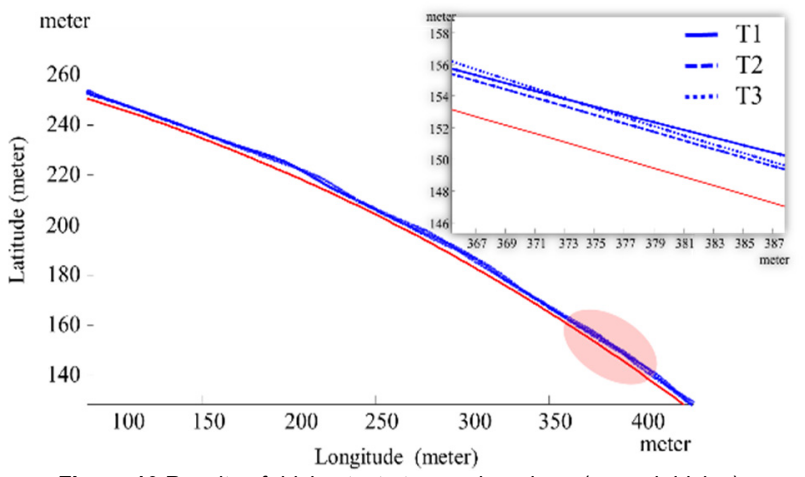

Figure 10 Results of driving test at curved roadway (normal driving)

Fig. 10 is showing detailed departure status. Between time $t=4$ and $t=4.25$ the state is started departing the earliest warning line from Lane I. The system allows to know an approximated position and direction of the vehicle driving. Uncertainty of positioning $(X v, Y v)$ is about entered lane departure area by unreached lane boundary due to uncertainties on the measurement sensor data.

From time $t=4.5$ to 4.75 the vehicle makes a departure to next lane area by real tire. It has exposed to risk of collision. After that, the test 1 and test 3 has returned to Lane I within short time, but test 2 has tried to crossing an earliest warning line of Lane II. The earliest warning line of next lane is not the latest warning line, but changing boundary line to next lane. From time $t=4.25$ to $t=5.75$ the alarming of test 1 and 3 is lasting, but test 2 is alarm off between time $t=4.9$ to $t=5.3$ due to lane changing status. According to these results, the returning pattern can find a lateral speed and degree of risk. 


\subsection{Result of Curve Section}

In the curve section, we demonstrated driving departure with low-speed consideration of safety. Like the preceding test, there are 2-types condition of driving test, which one is normal driving and another one is departure driving. Because driving condition is not particularly good, departure driving status absolutely depart from warning line.

Then, the results of trajectory have occasionally occurred departing to next lane when the vehicle departs warning line or recoveries again to original lanes.

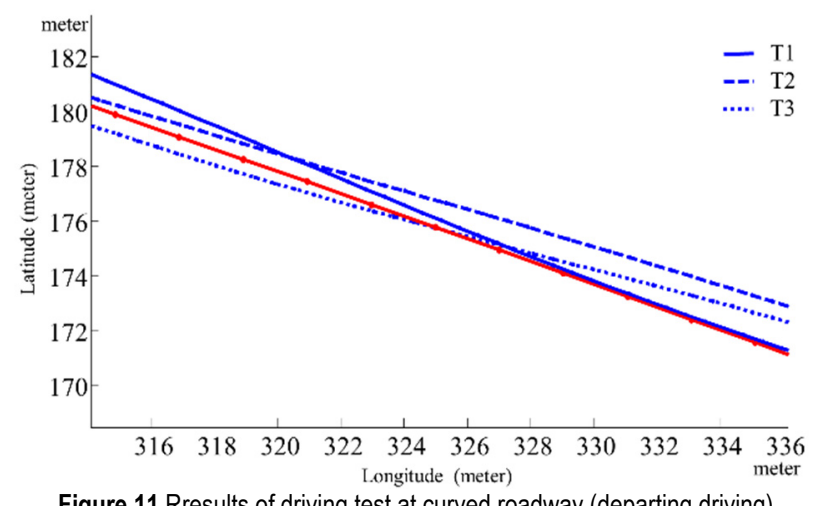

Figure 11 Rresults of driving test at curved roadway (departing driving)

Fig. 11 is shown a result of normal driving for false alarm. As shown a result of test trajectory, which is roughly far from lane boundary of curved roadway due to the fact that risk of curved roadway at high-speed in the two-lanes. Latitude and longitude coordinate have transformed by above Bezier curve using points of lane segments. Vehicle trajectory at curved road that can be observed a lane boundary line that are expressed by the GIS red line and three test lines with blue color (line, dotted, and dashed).

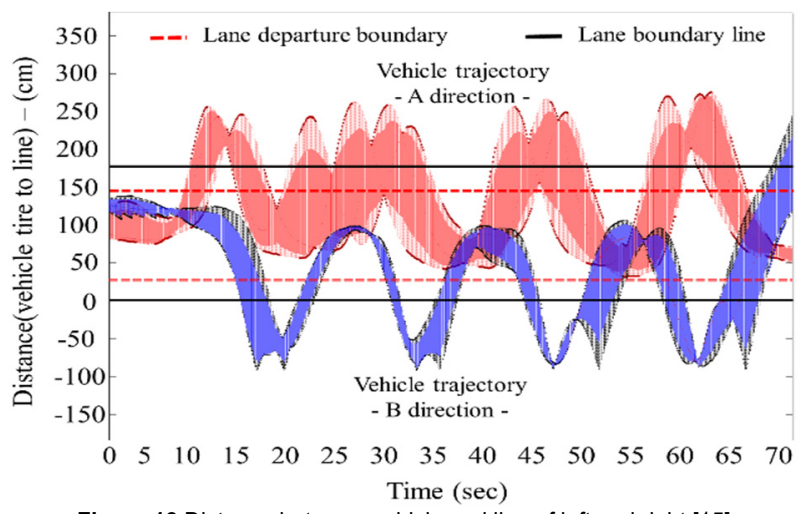

Figure 12 Distance between vehicle and line of left and right [15]

As with the preceding result, Fig. 12 shows the distance between vehicle and line (i.e., the vehicle drive curve section against updating GIS line). Fig. 13 is showing detailed results of distance between vehicle tire and the lane departure boundary.

A distribution of relative distance is the larger than straight driving. Normal driving is presented as a position at between $0.9-1.3 \mathrm{~m}$ from the boundary line; when the vehicle departs from this range, the blue and red blocks cross the lane departure boundary line at each interval.

These results have shown the suggested lane departure algorithm has been properly applied, even with curved road. Fig. 13 is showing when a vehicle happens crossing the earliest warning line.

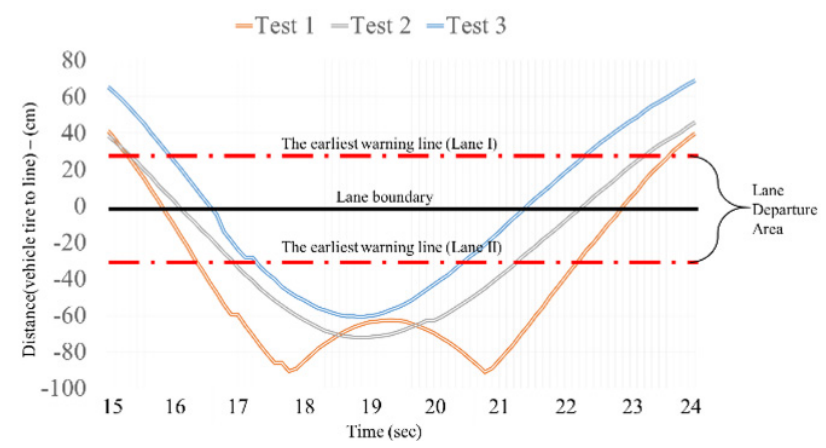

Figure 13 Vehicle trajectory when departing lane boundary

Approximately time $t=15.5$ starts lane departure, but their trajectory has taken effect to cross the earliest warning line of Lane II due to risk of collision to crash barriers. In the case of test 1 , between time $t=17.8$ to time $t=20.8$ its result of parabola is the opposite direction. Because lane boundary transformed by Bezier curve is overlapped a section to two curved lines.

\section{CONCLUSION}

This article presents a lane departure algorithm using DGPS sensor based on lane information obtained from GIS. As like previously research, a dynamic efficiency test is shown that positioning level is under $20 \mathrm{~cm}$ by transmitting RTCM message in the WAVE communication.

Furthermore, detecting algorithm has implemented each environment condition as straight and curved roadway, which has utilized compartmental area through classification method of roadway type and finding the intersection points.

In the straight section, vehicle lateral speed and direction angle of vehicle has offered help to compute distance between vehicle tire and lane boundaries. Lane elements as a reference line that are linked first point P0 and the intersection point $\mathrm{P}_{N}$ for linearized line has fitted lane boundary, which has matched each lane boundary of segment. In the curved section, lane segments updated by Bezier curve arithmetic has overlapped that area to share. The updated lane segments have created more 10-times by connecting 3-points segments. These segments can reduce error through comparison with real lane segments.

There is a possibility to analysis for characteristic vehicle travelling based on lane boundary using positioning data.

\section{Acknowledgment}

This work was supported by the National Research Foundation of South Korea funded by the Ministry of Education (No. 2019R1A2C2010195) and by the Ministry of Science and ICT of South Korea under the ICT Consilience Creative program (IITP-2019-2015-0-00742) supervised by the Institute for Information \& communications Technology Planning \& Evaluation. It was also supported by Ministry of Trade, Industry and Energy under Robot Industrial Core Technology Development Project program (K_G012000921401) supervised by the KEIT. 


\section{REFERENCES}

[1] Intelligent Transport System. Lane Departure Warning Systems-Performance requirements and test procedures. ISO 17361, 2007

[2] Jung, H., Lee, Y., Kang, H., \& Kim, J. (2009). Sensor fusionbased lane detection for LKS+ ACC system. International Journal of automotive technology, 10, 219-228. https://doi.org/10.1007/s12239-009-0026-0

[3] Kim, M. W., Moon, S. C., Joo, D. N., \& Lee, S. G. Matching GIS Lane Data with Vehicle position using camera image. Transactions of the Korean society of automotive engineers, 22, 40-47. https://doi.org/10.7467/KSAE.2014.22.7.040

[4] Leng, Y. C. \& Chen, C. L. (2010). Vision-based lane departure detection system in urban traffic scenes. Control Automation Robotics and Vision (ICARCV), 1875-1880. https://doi.org/10.1109/ICARCV.2010.5707817

[5] Moon, S. C., Kim, M. W., Joo, D. N., \& Lee, S. G. (2013). GPS-RTK Solution using WAVE Communication for vehicle. 20th ITS World Congress.

[6] Moon, S. C., Lee, S. G., \& Kim, D. H. Classification of roadway type based on roadway characteristics using RCCC. International Journal of Applied Engineering Research, 10, 27587-27592.

[7] Moon, S. C., Lee, S. G., Kim, J. J., \& Kim, B. S. (2012). Detection Lane Departure based on GIS using DGPS. Transactions of the Korean Society of Automotive Engineers, 20, 16-24. https://doi.org/10.7467/KSAE.2012.20.4.016

[8] Moon, S. C. A study on detection lane departure using DGPS/RTK based on GIS in Highway Straight section. Kyung Hee University Master's thesis.

[9] Moon, S. C., Lee, S. G., Kim, M. W., \& Joo, D. N. Assessment and reliability validation of lane departure assistance system based on DGPS-GIS using camera vision. Transaction of the Korean Society of Automotive Engineers, 22, 49-58. https://doi.org/10.7467/KSAE.2014.22.6.049

[10] Ozguner, U., Stiller, C., \& Redmill, K. (2007). System for safety and autonomous behavior in cars: The DARPA Grand Challege experience. Proceedings-IEEE, 95(397). https://doi.org/10.1109/JPROC.2006.888394

[11] Salomon, D. Curves and surfaces for computer graphics. Springer Science and Business Media.

[12] Skog, I. \& Handel, P. (2009). In-car positioning and navigation technologies-A survey. IEEE Transactions on intelligent Transportation Systems, 10, 4-21. https://doi.org/10.1109/TITS.2008.2011712

[13] Southall, B. \& Taylor, C. J. (2001). Stochastic road shape estimation. IEEE International Conference ICCV, 205-212.

[14] Toledo-Moreo, R. \& Zamora, M. A. IMM-based lanechange prediction in highways with low-cost GPS/INS IEEE Transactions on Intelligent Transportation Systems, 10, 180-185. https://doi.org/10.1109/TITS.2008.2011691

[15] Weon., I. S. \& Lee, S. G. (2019). Lane departure algorithm based on classification roadway type using DGPS/GIS. International Journal of Smart Home, 13(1), 1-6. https://doi.org/10.21742/ijsh.2019.13.1.01

[16] Hashim, R., Sibghotulloh Ikhmatiar, M., Miswan Surip, M. K., \& Tutut, H. (2011). Mosque Tracking on Mobile GPS and Prayer Times Synchronization for Unfamiliar Area. International Journal of Future Generation Communication and Networking, 4(2), 37-48.

[17] Lili, A., Wenham, X., Yancheng, L., \& Youtao, Z. (2016). A GPS Positioning Method for Underwater Targets Using Dual Acoustic Devices. International Journal of Future Generation Communication and Networking, 9(1), 115-126. https://doi.org/10.14257/ijfgen.2016.9.1.11
[18] Soham, S., Deabsree Chanda, S., Biswas, S., \& Partha Pratim, S. (2014). A Novel Approach to Identify a Fraud Website Using Android Smartphone under the Collaborative Frameworks of QR Codes and GPS and Motion Parameters of the User. International Journal of Security and Its Applications, 8(5), 161-184. https://doi.org/10.14257/ijsia.2014.8.5.16

[19] Yuyu, Y., Lu, C., Yueshen, X., Jian, W., He, Z., \& Zhida, M. (2019). QoS Prediction for Service Recommendation with Deep Feature Learning in Edge Computing Environment. Mobile Networks and Applications. https://doi.org/10.1007/s11036-019-01241-7

[20] Yuyu, Y., Fangzheng, Y., Yueshen, X., Lifeng, Y., \& Jinglong, M. (2017). Network Location-Aware Service Recommendation with Random Walk in Cyber-Physical Systems. Sensors, 17(9), 2059. https://doi.org/10.3390/s17092059

\section{Contact information:}

\section{Ihn-Sik WEON, PhD}

Incheon International Airport Corporation,

47, Gonghang-ro 424 beon-gil, Jung-gu, Incheon, Republic of Korea E-mail: innsik@airport.kr

\section{Soon-Geul LEE, PhD, Professor}

(Corresponding author)

Dept. of Mechanical Engineering, Kyung Hee University, 1732 Dukyoungdaero, Kiheing-gu, Yongin 17104, Korea E-mail: sglee@khu.ac.kr

\section{Soo-Ho WOO}

Dept. of Mechanical Engineering, Kyung Hee University, 1732 Dukyoungdaero, Kiheing-gu, Yongin 17104, Korea E-mail: woosh828@naver.com 